\title{
A Linear Algebra Approach To Synchronizing Automata
}

\author{
by
}

Fredrick C. Arnold, B. M.

\author{
A thesis submitted to \\ the Faculty of Graduate Studies and Research \\ in partial fulfillment of
}

\author{
Master of Science \\ School of Mathematics and Statistics \\ Ottawa-Carleton Institute of Mathematics and Statistics
}

\author{
Carleton University \\ Ottawa, Ontario, Canada \\ December 2004 \\ (C) copyright \\ 2004, Fredrick C. Arnold
}




$\begin{array}{ll}\begin{array}{l}\text { Library and } \\ \text { Archives Canada }\end{array} & \begin{array}{l}\text { Bibliothèque et } \\ \text { Archives Canada }\end{array} \\ \begin{array}{l}\text { Published Heritage } \\ \text { Branch }\end{array} & \begin{array}{l}\text { Direction du } \\ \text { Patrimoine de l'édition }\end{array} \\ \begin{array}{l}\text { 395 Wellington Street } \\ \text { Ottawa ON K1A ON4 } \\ \text { Canada }\end{array} & \begin{array}{l}\text { 395, rue Wellington } \\ \text { Ottawa ON K1A ON4 } \\ \text { Canada }\end{array}\end{array}$

Your file Votre référence ISBN: 0-494-00774-5

Our file Notre référence

ISBN: 0-494-00774-5

NOTICE:

The author has granted a nonexclusive license allowing Library and Archives Canada to reproduce, publish, archive, preserve, conserve, communicate to the public by telecommunication or on the Internet, loan, distribute and sell theses worldwide, for commercial or noncommercial purposes, in microform, paper, electronic and/or any other formats.

The author retains copyright ownership and moral rights in this thesis. Neither the thesis nor substantial extracts from it may be printed or otherwise reproduced without the author's permission.
AVIS:

L'auteur a accordé une licence non exclusive permettant à la Bibliothèque et Archives Canada de reproduire, publier, archiver, sauvegarder, conserver, transmettre au public par télécommunication ou par l'Internet, prêter, distribuer et vendre des thèses partout dans le monde, à des fins commerciales ou autres, sur support microforme, papier, électronique et/ou autres formats.

L'auteur conserve la propriété du droit d'auteur et des droits moraux qui protège cette thèse. $\mathrm{Ni}$ la thèse ni des extraits substantiels de celle-ci ne doivent être imprimés ou autrement reproduits sans son autorisation.
In compliance with the Canadian

Privacy Act some supporting forms may have been removed from this thesis.

While these forms may be included in the document page count, their removal does not represent any loss of content from the thesis.
Conformément à la loi canadienne sur la protection de la vie privée, quelques formulaires secondaires ont été enlevés de cette thèse.

Bien que ces formulaires aient inclus dans la pagination, il n'y aura aucun contenu manquant. 


\begin{abstract}
Čemý's conjecture is a 40 years old open problem concerning the synchronizing of finite automata. Namely, it proposes an upper bound on the length of minimal synchronizing words for finite automata in terms of the number of states. So far, the conjecture has resisted proof except for some special cases of automata. We consider the following special cases: weakly orientable automata, Eulerian automata, and irreducible permutation automata.
\end{abstract}




\section{Acknowledgements}

I would like to thank the people who have helped in their own way while I've been writing my thesis. Firstly, my friend Terri, for helping me with LaTeX tips when I wasn't sure how to do something properly. Secondly, my parents and my friends Gail and Susan, for the support and advice they offered me. And finally, Professor Steinberg, for all his helpful suggestions and explanations. 


\section{Contents}

Abstract $\quad$ iii

Acknowledgements $\quad$ iv

Chapter 1. Introduction 1

Chapter 2. Introduction to Automata 3

Chapter 3. Auxiliary Automata 10

Chapter 4. Weakly Orientable Automata 15

Chapter 5. Linearization of Automata 27

Chapter 6. Eulerian Automata 35

Chapter 7. Irreducible Permutation Automata 38

$\begin{array}{lll}\text { Chapter 8. Conclusion } & 46\end{array}$

$\begin{array}{ll}\text { Bibliography } & 47\end{array}$ 


\section{CHAPTER 1}

\section{Introduction}

It has happened to us all at some point, we're driving along in a city with our car trying to follow a set of directions to get somewhere when we take a wrong turn or drive past one of the turns. This can often lead to us getting lost while we try to figure out how to get back on track since there are no directions from where we ended up to where we wanted to go. It would be much easier to find our way around if there were a set of directions that would take us to our desired destination no matter where we started. It is this type of problem that Černý considered in 1964. Except he considered machines called automata instead of a city road map. But, the idea is essentially the same and he used the word synchronizing to describe these sets of directions, and maps that had such directions. To use our analogy, Černý produced, for each $n \in \mathbb{N}$, an example of a road map with $n$ intersections that had minimal synchronizing directions with $(n-1)^{2}$ steps to follow (the construction to follow in Chapter 2) and conjectured that these maps represented the worst possible case, that is, the shortest synchronizing directions for every synchronizing map with $n$ intersections can not have more than $(n-1)^{2}$ steps. This conjecture has never been proven in general, and only special cases have been considered in the literature $[1],[6],[7],[8],[15]$.

There are many practical applications where this sort of problem is of interest. For instance, the handling of parts by automated machines when dealing with tasks such as part feeding, loading, assembly, and packing [11],[12]. To elaborate upon this, let us consider the case when mechanical parts are shipped in boxes to a manufacturing site for assembly. These parts will need to be sorted and oriented properly before assembly can begin. For simplicity, we shall only consider the orientation process for 
a certain type of part where a conveyor belt with a series of obstacles is used to adjust the orientation of the parts. The problem becomes how many obstacles do we need and how do we set them up so that the parts end up in the desired orientation.

Another example involves biocomputing where a simulation of a solution consisting of many identical automata has been produced using DNA molecules [2],[3]. These automata can then be used in parallel on different inputs and hence will likely end up in different states. Once these automata have finished their work, it would be useful to be able to reset all the automata to a predetermined start state. In this case, the problem becomes how to construct a DNA molecule whose nucleotide sequence will reset these automata and adding a sufficient amount of them to the solution [2],[3].

In this thesis, we begin with an introduction to automata and Černý's examples in Chapter 2. After that, we shall introduce auxiliary automata that can be used to prove a cubic upper bound on the length of a synchronizing word in Chapter 3 . Returning to the automata discussed in Chapter 2, we put certain restrictions on the input alphabet and prove that Černý's conjecture holds under this restriction in Chapter 4. Then, we shall discuss how to interpret an automaton in terms of linear algebra concepts in Chapter 5. And, using these concepts, we shall look at Eulerian automata in Chapter 6 and circular automata with a prime number of states in Chapter 7. These last two chapters form our contribution to the problem by introducing a unified linear algebra approach to solving this type of problem and presenting a new theorem in Chapter 7 that proves a more general result than the previously established result for circular automata with a prime number of states. 


\section{CHAPTER 2}

\section{Introduction to Automata}

We start by introducing the concept of an alphabet. An alphabet $A$ is a finite non-empty set. A word (or string) over $A$ is a finite sequence $a_{1} a_{2} \ldots a_{n}$ where each $a_{i} \in A$ and the length of this sequence is $n$. We also include the empty string, denoted by $\varepsilon$. We let $A^{*}$ denote the set of all words over $A$ (including $\varepsilon$ ). A subset $L$ of $A^{*}$ is called a language. To put this into context, we can think of $A$ as the set of all words in a dictionary. Then we define $L \subseteq A^{*}$ as the finite sentences which are grammatically correct.

In this thesis we will be considering (finite state) automata. This automaton considers of a finite set of internal states $Q$ and a finite alphabet $A$. The alphabet represents the input to this automaton that can be strung together to form words. Also, each letter in these words will successively change the internal state depending on the current state and the input letter.

DEFINITION 2.1. An automaton consists of a finite set of states $Q$, usually represented by $\{1, \ldots, n\}$, a finite input alphabet $A$, and a transition function $\delta: Q \times A \rightarrow Q$ that defines the transition from one state $q$ into another state $q^{\prime}=\delta(q, a)$ when the automaton receives the input symbol a. We often denote the automaton by $(Q, A)$, leaving the transition function understood.

EXAMPLE 2.2. Consider the non-cyclic group $G=\{I, a, b, a b\}$, where $a^{2}=b^{2}=I$ and $a b=b a$. We want to construct a finite automaton to compute in $G$, with a two letter alphabet $A=\{a, b\}$. When we input a word (e.g. abbaaab), we want to find the result of the product in $G$ (in this case b). Since we have four elements in $G$, we will have 4 states in our automaton and we will use the elements as the labels for the 
states.

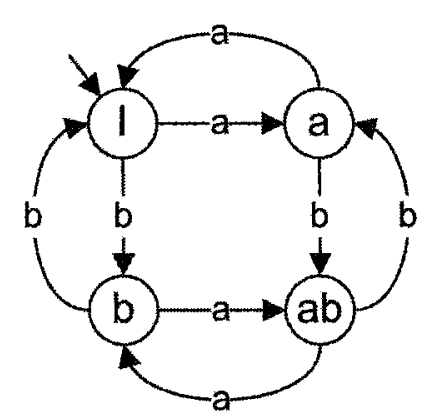

The labelled directed edges in the diagram represent the transition function that determines how the input letter changes the current state of the automaton. This diagram is called the transition graph of the automaton where the unlabelled arrow to state $I$ denotes an initial state.

We shall use the notation $q \cdot a$ instead of $\delta(q, a)$. We can extend this to words as follows:

- $q \cdot \varepsilon=q$

- $q \cdot u a=(q u) a$ for $u \in A^{*}, a \in A$.

To be able to recognize a language, we can construct an automaton called an accepter. An accepter is an automaton that also has an initial state $q_{0} \in Q$ and a set of final states $F \subseteq Q$. We shall denote this automaton with the tuple $M=$ $\left(Q, A, \delta, Q_{0}, F\right)$. The, the language of this accepter consists of the words that take the initial state to one of the final states. More precisely, $L(M)=\left\{w \in A^{*} \mid q \cdot w \in F\right\}$ is the language of this accepter.

EXAMPLE 2.3. An example of an accepter is as follows,

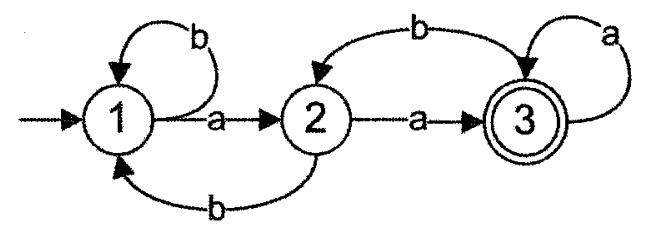

where $q \stackrel{a}{\longrightarrow} q^{\prime}$ if $q^{\prime}=q \cdot a$. Also, the double circle on state 3 means that it is a final state. 
An automaton $(Q, A)$ is said to be synchronizing if there is a word $w$ such that $q \cdot w=q^{\prime} \cdot w$ for all $q, q^{\prime} \in Q$, i.e. regardless of the state of the automaton, $w$ leads to the same state. This $w$ is called a synchronizing (or reset) word in $(Q, A)$. But, it is often easier to work backwards from a single state and try to get all the states. This motivates defining, for $w \in A^{*}, q w^{-1}=\left\{q^{\prime} \mid q^{\prime} w=q\right\}$. We also define for $S \subseteq Q$, $w \in A^{*}, S w=\{q w \mid q \in S\}$.

Our strategy for finding synchronizing words will then be to show that for a given non-empty, proper subset $S \subseteq Q$, we can find a word $u \in A^{*}$ such that $\left|S u^{-1}\right|>|S|$ where $S u^{-1}=\{q \in Q \mid q \cdot u \in S\}$. In other words, given a proper subset $S$ we want to find a word $u$ - not necessarily the entire synchronizing word - whose inverse when applied to $S$ will give us a new set $S u^{-1}$ that has size at least one greater than $S$. Then we will be able to find a synchronizing word by starting with a one element set and expanding repeatedly. If $u$ can always be chosen to have size at most $k$, then we can construct a synchronizing word of size at most $1+(n-2) k$. Indeed, we can expand a one element set with a single letter and then we have to expand $n-2$ more times using our bound $k$. In particular, if $k=n=|Q|$, then we get $1+(n-2) n=(n-1)^{2}$.

Proposition 2.4. Let $(Q, A)$ be an automaton where any two states can be connected by a directed path. If the automaton is synchronizing, we can find a synchronizing word to go to any state.

Proof. Since the automaton is synchronizing, there exists a word $w \in A^{*}$ that takes every state in $(Q, A)$ to a single state $q \in Q$. But, since any two states can be connected in $(Q, A)$ by a directed path, for any $q^{\prime} \in Q$ there is a word $u \in A^{*}$ that takes $q$ to $q^{\prime}$. Clearly, the word $w u$ synchronizes the automaton to $q^{\prime}$.

Therefore, under the conditions of Proposition 2.4, any non-empty subset of states of a synchronizing automaton can be expanded.

CONJECTURE 2.5 (Černý's conjecture for automata). Every synchronizing automaton with $n$ states has a shortest synchronizing word with length at most $(n-1)^{2}$. 
DEFINITION 2.6. A circular automaton is an automaton that contains an input letter that cyclically permutes the states.

THEOREM 2.7 (Černý $[5])$. Let $(Q, A)$ be the circular automaton with $Q=\{1, \ldots, n\}$ and $A=\{a, b\}$ defined as follows. For any $q, q^{\prime} \in Q, q a=q+1(\bmod n), q^{\prime} b=q^{\prime}$ if $q^{\prime} \neq 2$, and $2 b=3$. Then the unique shortest synchronizing word for $(Q, A)$ is $\left(b a^{n-1}\right)^{n-2} b$, which has length $(n-1)^{2}$.

Proof. Our automaton is constructed as shown,

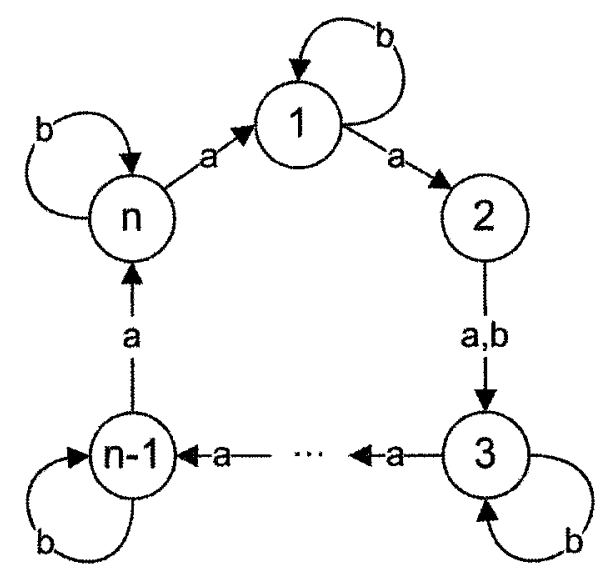

Notice that if we're at a state $x$ in the automata, then

$$
x b a^{n-1}= \begin{cases}x-1(\bmod n), & \text { if } x \neq 2 \\ 2, & \text { if } x=2 .\end{cases}
$$

We remark that $1-1(\bmod n)$ is $n$. As a consequence of this,

$$
x\left(b a^{n-1}\right)^{n-2}= \begin{cases}3, & \text { if } x=1 \\ 2, & \text { else. }\end{cases}
$$

Thus, $\left(b a^{n-1}\right)^{n-2} b$ is a synchronizing word. Let $w$ be a minimal length synchronizing word for $(Q, A)$. Then we notice that two consecutive $b$ s will not produce anything different than a single $b$, and thus will not occur in a minimal synchronizing word.

If we consider all $n$ states of the automaton, we can see that $b$ will collapse the number of states as $b$ will take state 2 to state 3 and take all other states to themselves. 
Thus, $w$ should begin and end with $b$, since $a$ will never collapse the number of states as it is just a permutation. Also, there should never be $n$ or more consecutive as since $a$ has order $n$.

DEFINITION 2.8. A segment is a set of consecutive states in circular order. (e.g. $n 12$ is a segment.) More precisely, if we view the states $1, \ldots, n$ arranged in a cycle, then a segment consists of a set of consecutive states on the circle.

DEFINITION 2.9. The left end of a segment is the left most state that appears in the segment in circular order. (e.g. 1 is the left end of 123)

For a segment $S \subseteq Z_{n}$, we observe that:

- $S a^{-1}=\{x-1(\bmod n) \mid x \in S\}$

- $S b^{-1}= \begin{cases}S \cup\{2\}, & \text { if the left end of } S \text { is } 3 ; \\ S \backslash\{2\}, & 2 \in S \text { and } 3 \notin S ; \\ S, & \text { else. }\end{cases}$

With these observations, it is easy to see that an inverse image of a segment is a segment (a rigorous proof for the more general situation appears in Chapter 4). Recall that we have two symbols in the alphabet: $a$ and $b$. It is clear that $a^{-1}$ will not change the size of a segment by the above. Also, recall that $b$ is never used twice consecutively in a minimal word. When $b^{-1}$ is used in a minimal word backwards, cases 2 and 3 above never occur since in the forward direction, we only use $b$ in a minimal word when it decreases the size of the image. Thus, the number of $b$ s must be $n-1$. Then putting together all that we know about $w$ so far, we're looking for a word of the form $\left(b a^{m_{i}}\right)^{k} b$ where $k$ has been found to be $n-2$ and $i=1, \ldots, k$. All that remains now is to show that each of the $m_{i}$ are equal to $n-1$.

Notice that we can increase the size of a segment with $b^{-1}$ only when the segment has state 3 as its left end. Let $S$ be such a segment. When we apply $b^{-1}$ to $S$, we will get a new segment $S^{\prime}$ that has state 2 as its left end. Then since $a^{-1}$ rotates $S^{\prime}$ counter-clockwise, we will need $n-1 a^{-1}$ s to get back to having state 3 as the left end. Thus, since we want $w$ to be the shortest synchronizing word, all the $m_{i}$ s must 
be $n-1$. Therefore, $w=\left(b a^{n-1}\right)^{n-2} b$.

We shall now introduce two variants of Črný's example, where one will be a special case where we can find a shorter synchronizing word and the other will not have a synchronizing word.

EXAMPLE 2.10. We construct our first variant as follows:

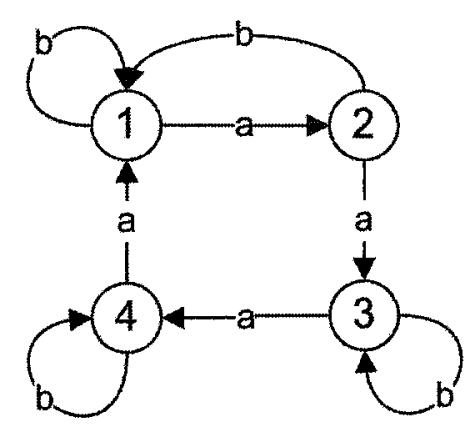

In this case, the shortest synchronizing word is babab which takes the automaton to state 1 and has length $5\left(<(4-1)^{2}=9\right)$. This is clearly the shortest such word since we know from Černy's example that we need 3 bs and at least one a between each pair of $b s$.

EXAMPLE 2.11. We construct our second variant as follows:

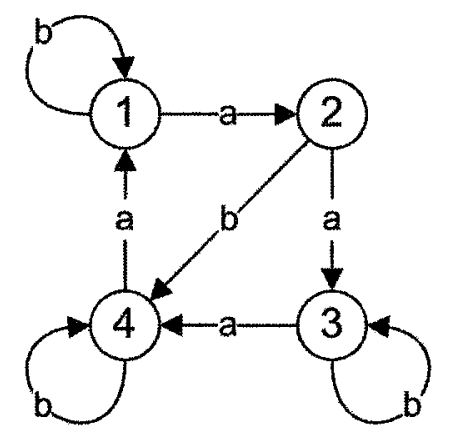

In this case, there is no synchronizing word but we shall leave our argument until Chapter 3 when we talk about auxiliary automata constructions.

Pin proved in $[\mathbf{1 5}]$ that any circular automaton with a prime number of states and a non-permutation input letter is synchronizing with a minimal synchronizing word of length at most $(n-1)^{2}$. We present a proof in Chapter 7 , proving in fact a more 
general result. Dubuc [6] proved Černýs conjecture holds for any circular automaton. Many cases of circular automata can also be handled by the technique of Chapter 4 due to [1]. 
CHAPTER 3

\section{Auxiliary Automata}

Consider the automaton $(Q, A)$. From this automaton, we define a new automaton, called the Exterior Power automaton $\bigwedge^{2}(Q, A)$.

DEFINITION 3.1. The Exterior Power automaton is an automaton where the states are 2-element subsets of $Q$. This means that the states are the unordered pairs of distinct elements of $Q$. We also add a new state $\infty$ that will be used to indicate that a pair has been collapsed. Since there are $\frac{|Q|(|Q|-1)}{2}$ such pairs, there are $\frac{|Q|(|Q|-1)}{2}+1$ states. Our alphabet $A$ does not change from that of $(Q, A)$, but we have a new set of transitions. They are as follows,

- for all $a \in A$, there exists $\{x, y\} \stackrel{a}{\longrightarrow}\{u, v\}$ if $x \stackrel{a}{\longrightarrow} u$ and $y \stackrel{a}{\longrightarrow} v$ in $(Q, A)$ and $u \neq v$.

- for all $a \in A$, there exists $\{x, y\} \stackrel{a}{\longrightarrow} \infty$ if $x \stackrel{a}{\longrightarrow} u$ and $y \stackrel{a}{\longrightarrow} u$ in $(Q, A)$.

LEMMA 3.2. A word $w \in A^{*}$ is a synchronizing word in $(Q, A)$ if and only if $(i, j) w=\infty$ for all $(i, j) \in \Lambda^{2}(Q, A)$.

Proof. A word $w \in A^{*}$ is a synchronizing word in $(Q, A)$ if and only if it takes all states in $Q$ to the same state $q \in Q$. Equivalently, $w$ is a synchronizing word if it collapses all unordered pairs of $(Q, A)$. More precisely, $q \cdot w=q^{\prime} \cdot w$ for all $q, q^{\prime} \in Q$ if and only if $\left\{q, q^{\prime}\right\} \stackrel{w}{\longrightarrow} \infty$ for all unordered pairs $\left\{q, q^{\prime}\right\}$.

EXAMPLE 3.3. Recall Example 2.10. Then we can construct the following Exterior Power automaton, 


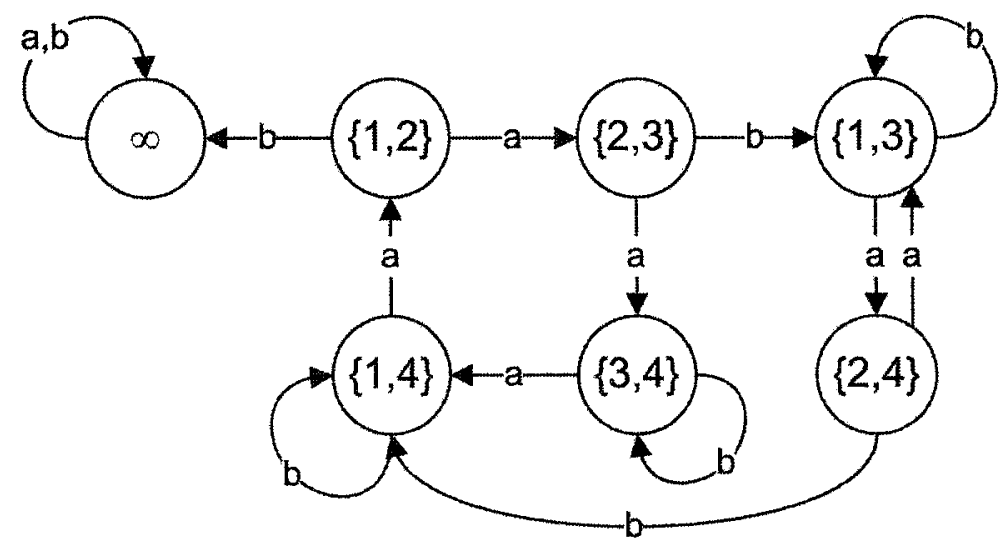

Notice that each unordered pair state is able to reach the $\infty$ state.

EXAMPLE 3.4. Recall Example 2.11. Then we can construct the following Exterior Power automaton,
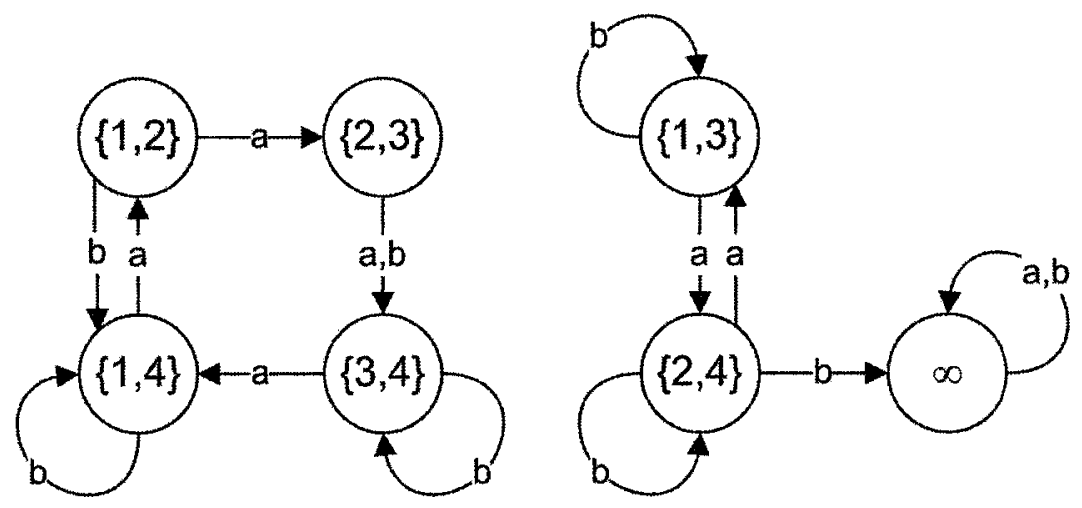

Unlike the previous example, there are clearly some unordered pair states that are unable to reach the $\infty$ state. Hence the automaton from Example 2.11 is not synchronizing.

We now construct a new automaton, called the Subset Construction $\Psi(Q, A)$ from $\bigwedge^{2}(Q, A)$. The states of $\Psi(Q, A)$ are all subsets of the states of $\bigwedge^{2}(Q, A)$ not containing $\infty$ (including $\emptyset$ ). As a result, this automaton has $2^{\frac{|Q|(|Q|-1)}{2}}$ states. The initial state is the state containing the set of all unordered pairs and the final state is the $\emptyset$ state. The alphabet is still $A$ from $(Q, A)$, but we have a new set of transitions as follows, 
- If $x=\left\{\left\{x_{i}, y_{i}\right\} \mid i \in I\right\}$, then $x \stackrel{a}{\longrightarrow} y$ where

$$
y=\left\{\left\{x_{i}, y_{i}\right\} a \mid i \in I,\left\{x_{i}, y_{i}\right\} a \neq \infty\right\}
$$

EXAMPLE 3.5. For the automaton

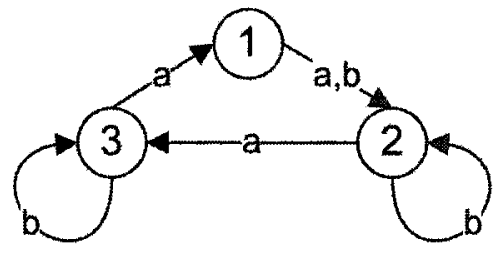

we can construct the following Subset Construction (with inaccessible states removed):

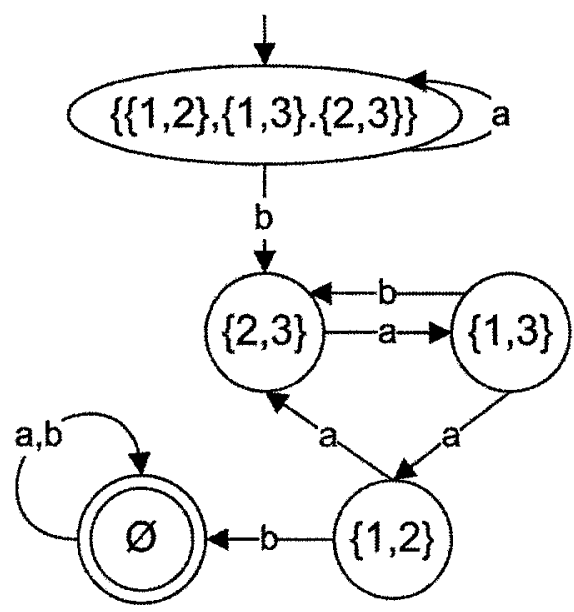

THEOREM 3.6. For an automaton $(Q, A)$, the language accepted by $\Psi(Q, A)$ is the language of synchronizing words. (This language may be empty.)

Proof. Let $X$ be the initial state of $\Psi(Q, A)$ and $w \in A^{*}$. We show by induction on the length of $w$ that

$$
X w=\{\{i, j\} w \mid i, j \in Q, i \neq j,\{i, j\} w \neq \infty\}
$$

If $w$ has length zero, then $w=\varepsilon$. So, $X \varepsilon=X$ and $\{i, j\} \varepsilon=\{i, j\}$ for all $\{i, j\}$. Thus (3.1) holds when the length of $w$ is zero. Suppose (3.1) holds for words of length $n \geq 0$. Let $w$ have length $n+1$. So, $w=v a$,where $v \in A^{*},|v|=n$, and $a \in A$. Then 


$$
X w=(X v) a=\{\{i, j\} v \mid\{i, j\} v \neq \infty\} \cdot a
$$

by induction. So,

$$
\begin{aligned}
X w & =\{(\{i, j\} v) a \mid(\{i, j\} v) a \neq \infty\} \\
& =\{\{i, j\} w \mid\{i, j\} w \neq \infty\}
\end{aligned}
$$

This completes the induction. Hence, in particular,

$$
\begin{aligned}
X w=\emptyset & \Leftrightarrow\{\{i, j\} w \mid\{i, j\} w \neq \infty\}=\emptyset \\
& \Leftrightarrow\{i, j\} w=\infty \text { for all }\{i, j\} \\
& \Leftrightarrow w \text { is a synchronizing word by Lemma 3.2. }
\end{aligned}
$$

Therefore, the language of $\Psi(Q, A)$ is precisely the language synchronizing words.

Thus, the language of synchronizing words of an automaton is regular. We shall now use the Exterior Power automaton to prove the cubic upper bound on synchronizing words $[\mathbf{1 6}]$. Our proof is more automata-theoretical than [16].

Proposition 3.7 (Salomaa $[\mathbf{1 6}]$ ). If $(Q, A)$ is synchronizing, then there exists a synchronizing word of length at most $1+(n-2) \frac{n(n-1)}{2}=\frac{n^{3}-3 n^{2}+2 n+2}{2}$, where $n=|Q|$.

Proof. We saw in Lemma 3.2 that $w$ is a synchronizing word precisely when it collapses all pairs. However, it suffices to show that $w$ collapses all pairs of the form $\{1, i\}, i=\{2, \ldots, n\}$. Indeed suppose that $w$ collapses all such pairs and $i \neq j$. Then $i \cdot w=1 \cdot w=j \cdot w$. Hence we only need to collapse these $n-1$ pairs.

Since $(Q, A)$ is synchronizing, some letter collapses at least two states. We may assume some $a \in A$ collapses $\{1,2\}$ by renumbering the states if needed. Then (setting $u_{2}=a$ ), we assume by induction that for $k \geq 2$ we can find, $u_{2} \ldots, u_{k} \in A^{*}$, each with length at most $\frac{n(n-1)}{2}$, such that $u_{2} \ldots u_{k}$ collapses $\{1,2\}, \ldots,\{1, k\}$. If $u_{2} \ldots u_{k}$ already collapses $\{1, k+1\}$, take $u_{k+1}=\varepsilon$. Otherwise $\{1, k+1\} u_{2} \ldots u_{k}=\{i, j\}$ in 
$\bigwedge^{2}(Q, A)$ for some $i, j \in Q$ where $i \neq j$.

The words collapsing $\{i, j\}$ are the words accepted by $\bigwedge^{2}(Q, A)$ with initial state $\{i, j\}$ and final state $\infty$. Since $(Q, A)$ is synchronizing, some word takes $\{i, j\}$ to $\infty$ by Lemma 3.2. The shortest such word never visits the same state of $\bigwedge^{2}(Q, A)$ twice. So, in the worst case, we visit all states on our way from $\{i, j\}$ to $\infty$. There are $\frac{n(n-1)}{2}+1$ such states. Since a word has length one less than the number of states it visits, we can find $u_{k+1} \in A^{*}$ with length at most $\frac{n(n-1)}{2}$ such that $\{i, j\} u_{k+1}=\infty$.

So, $u_{2} \ldots u_{k} u_{k+1}$ still collapses $\{1,2\}, \ldots,\{1, k\}$, but now it collapses $\{1, k+1\}$ as well. In particular, $w=u_{2} \ldots u_{n}$ collapses $\{1,2\}, \ldots,\{1, n\}$. Since $\left|u_{2}\right|=1, w$ is a synchronizing word with length at most

$$
1+\sum_{i=3}^{n} \frac{n(n-1)}{2}=1+(n-2) \frac{n(n-1)}{2} .
$$

Therefore, we have obtained our desired bound. 
CHAPTER 4

\section{Weakly Orientable Automata}

In this chapter, we think of $Q=\{1, \ldots, n\}$ as a cycle oriented clockwise, e.g.

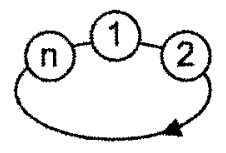

That is, if $m \equiv k+1(\bmod n)$, then we consider $m$ the (immediate) successor of $k$ and $k$ the (immediate) predecessor of $m$. A sequence $i_{1}, \ldots, i_{k}$ is said to be in circular order if it follows the order of the cycle, that is $i_{1}, \ldots, i_{k}$ contains at most one index $j$ such that $i_{j}>i_{j+1}$, where $j$ is taken $\bmod n$. A transformation $\alpha: Q \rightarrow Q$ is called orientation preserving if $1 \alpha, \ldots, n \alpha$ is in circular order; $\alpha$ is called orientation reversing if $n \alpha, \ldots, 1 \alpha$ is in circular order.

An automaton $(Q, A)$ is said to be weakly orientable if each letter $a \in A$ acts as an orientation preserving or orientation reversing transformation. We remark that if $\alpha, \beta$ are both orientation preserving or both orientation reversing, then $\alpha \beta$ is orientation preserving, while if one is orientation preserving and the other is orientation reversing, then $\alpha \beta$ is orientation reversing. Thus, in a weakly orientable automaton, each word also acts as either an orientation preserving or orientation reversing transformation. Examples of weakly orientable automata are Černý's examples in Theorem 2.7.

EXAMPLE 4.1. Let

$$
a=\left(\begin{array}{llll}
1 & 2 & 3 & 4 \\
2 & 3 & 4 & 1
\end{array}\right) .
$$

Since a encodes the sequence $2,3,4,1$, a is clearly orientation preserving. This can also be interpreted via the following diagram: 


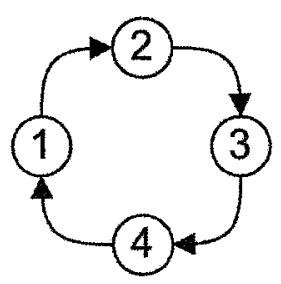

Namely, if we write the bottom line in the description of a as a cycle, then a is orientation preserving if we can start at one of these vertices and find an non-decreasing path until we return to where we started. In this case, we must start at 1.

EXAMPLE 4.2. Let

$$
b=\left(\begin{array}{llll}
1 & 2 & 3 & 4 \\
3 & 2 & 2 & 1
\end{array}\right) .
$$

Since $b$ encodes the sequence $3,2,2,1, b$ is clearly orientation reversing. As above, this can also be interpreted via the following diagram:

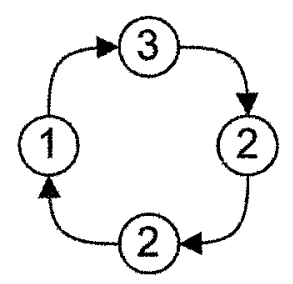

Namely, if we write the bottom line in the description of $b$ as a cycle, then $b$ is orientation reversing if we can start at one of these vertices and find a non-increasing path until we return to where we started. In this case, we must start at 3 .

EXAMPLE 4.3. A typical example that is neither orientation preserving nor reversing is when a letter encodes a sequence like $i, j, i, k$, where $j, k \neq i$. For instance, let

$$
c=\left(\begin{array}{llll}
1 & 2 & 3 & 4 \\
2 & 3 & 2 & 1
\end{array}\right) .
$$

The corresponding diagram is as follows: 


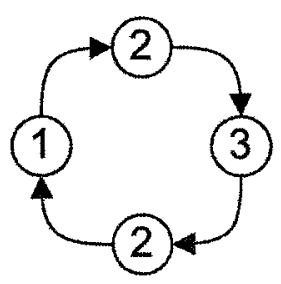

Notice in the diagram there is neither a non-increasing nor a non-decreasing path of length 4 .

EXAMPLE 4.4. Let $(Q, A)$ be an automaton with 4 states with input alphabet $A=$ $\{a, b\}$, where $a$ is taken from Example 4.1 and $b$ is taken from Example 4.2.

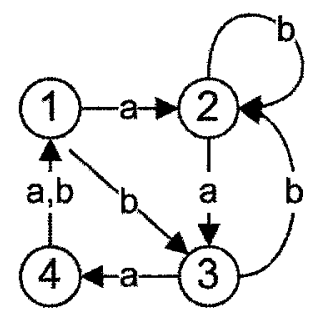

This is an example of a weakly orientable automaton with a shortest synchronizing word bbb. The reasoning is exactly the same as in Example 2.10.

Recall that a segment is a set of consecutive states in circular order. In this chapter, we use $[i, j]$ to denote the segment with left end $i$ and right end $j$. By an interval $(i, j)$, we mean an arbitrary pair of states in $Q$. We say that $(i, j)$ represents the segment $[i, j]$. With this definition, we see that $(2,1),(3,2), \ldots,(n, n-1),(1, n)$ are different intervals even though they all represent the set $Q$. However, any proper segment of $Q$ is represented by a unique interval. We assume from now on $(Q, A)$ is weakly orientable.

LEMMA 4.5. If $S \subseteq Q$ is a segment, then $S w^{-1}$ is a segment.

ProOf. If $S w^{-1}$ is $Q$, we have nothing to prove. If $S w^{-1}$ is a proper subset of $Q$, then so is $S$. Let $(i, j)$ be the unique interval representing $S$ and let $\left[k_{1}, k_{2}\right]$ be a maximal segment such that $\left[k_{1}, k_{2}\right] \subseteq S w^{-1}$. Since $\left[k_{1}, k_{2}\right]$ is maximal, if we choose $l_{1}, l_{2} \in Q$ such that $l_{1}$ immediately precedes $k_{1}$ and $k_{2}$ immediately precedes $l_{2}$ in the 
circular order, then we have that $l_{1} w, l_{2} w \notin S$. If $\left[k_{1}, k_{2}\right] \neq S w^{-1}$, there exists an element $k_{3} \in Q$ between $l_{2}$ and $l_{1}$ such that $k_{3} w \in S$.

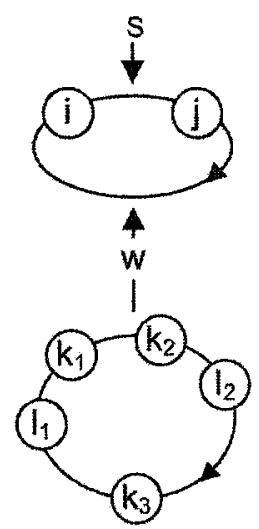

Observe that the sequence $k_{1}, k_{2}, l_{2}, k_{3}, l_{1}$ is in circular order and maps to $k_{1} w, k_{2} w, l_{2} w, k_{3} w, l_{1} w$ under $w$. We show that neither $k_{1} w, k_{2} w, l_{2} w, k_{3} w, l_{1} w$ nor is its reversal are in circular order. There are 3 possibilities:

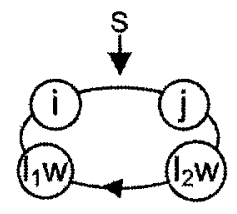

or

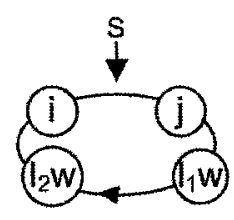

or

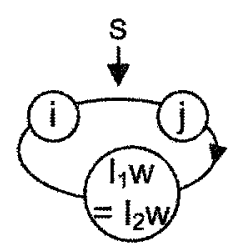

We just handle the first case, since the others are similar. Since $k_{3} w \in S$ and so it is not between $l_{2} w$ and $l_{1} w$, we see that $w$ can not be orientation preserving. Since $k_{2} w, k_{1} w \in S$ and so they are not between $l_{2} w$ and $l_{1} w$, we have that $l_{2} w, k_{2} w, k_{1} w, l_{1} w$ is not in circular order, so $w$ is not orientation reversing. This is a contradiction. 
For each weakly orientable automaton $(Q, A)$, we construct its interval automaton $\mathcal{I}(Q, A)$ where the states are all intervals over $\{1, \ldots, n\}$ and we add an additional state $\infty$ that is a sink state. Since there are $n^{2}$ such intervals, there are $n^{2}+1$ states. Our alphabet $A$ does not change from the $(Q, A)$, but we have a new set of transitions. They are as follows.

Let $(i, j)$ be an interval and $a \in A$. Let $k=i a$ and $l=j a$. We have two cases: $k \neq l$ or $k=l$.

- If $k \neq l$, define $(i, j) a= \begin{cases}(k, l), & \text { if } a \text { is orientation preserving; } \\ (l, k), & \text { if } a \text { is orientation reversing. }\end{cases}$

- If $k=l$, define $(i, j) a= \begin{cases}(k, k), & \text { if } h \stackrel{a}{\longrightarrow} k \text { for all } h \in[i, j] ; \\ \infty, & \text { else. }\end{cases}$

We shall prove $w \in A^{*}$ is a synchronizing word if and only if there exists $(i, j)$ representing all of $Q$ such that $(i, j) w=(k, k)$ for some $k$.

EXAMPLE 4.6. The following is the interval automaton for the weakly oriented automaton in Example 4.4 .

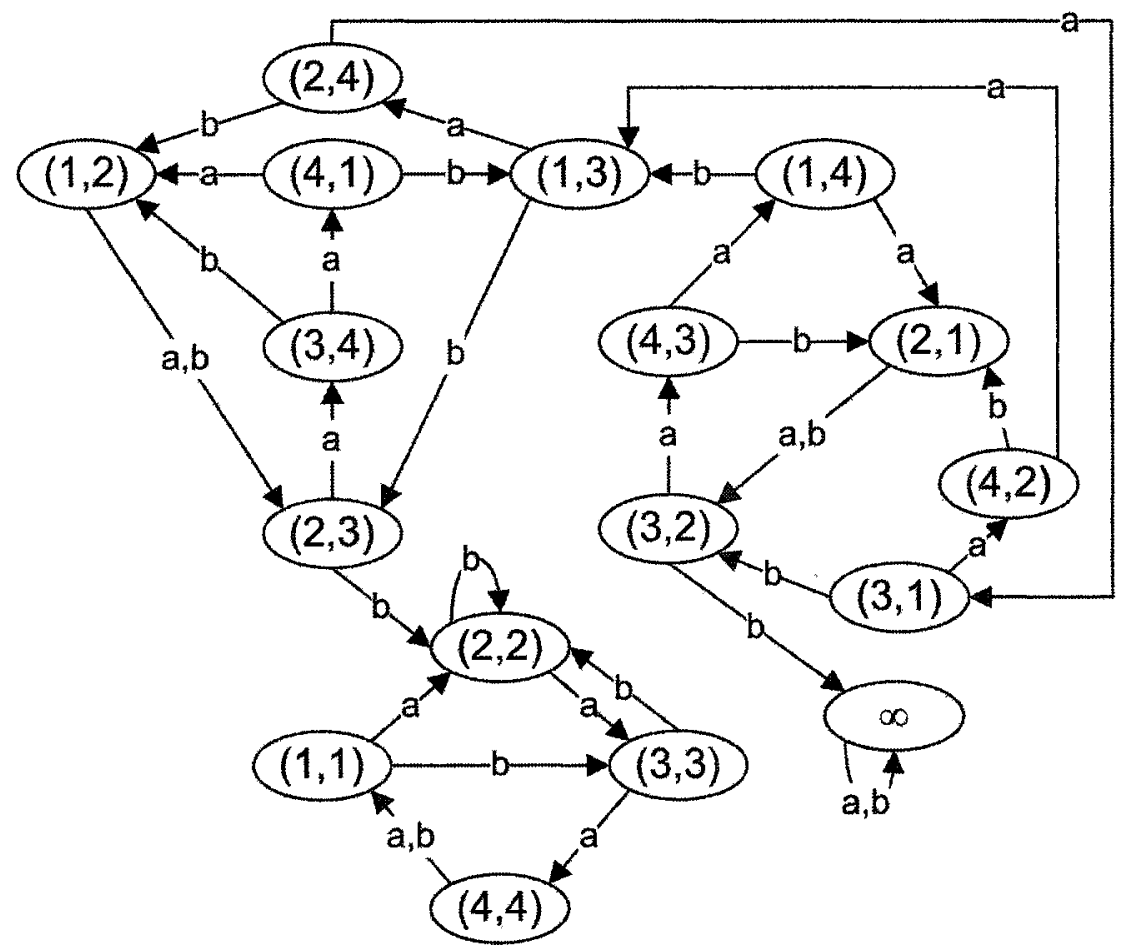

Notice that bbb takes $(1,4)$ to $(2,2)$ but it doesn't take any of the other intervals 
representing $\{1,2,3,4\}$ to $(2,2)$.

For the interval automaton, we need the following observation.

LEMMA 4.7. If $a \in A$ and $(i, j)$ is an interval such that $(i, j) a=\infty$ and $i a=k$, then $(j, i) a=(k, k)$.

Proof. Recall that $(i, j) a=\infty$ means that $i a=j a=k$ but $h a \neq k$ for some $h$ between $i$ and $j$. Also, if $(j, i) a=\infty$, there exists some $g$ between $j$ and $i$ such that $g a \neq k$.

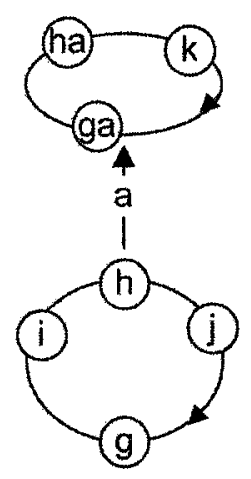

The sequence $i, h, j, g$ is in circular order and maps to $k, h a, k, g a$. . But, as we've seen in Example 4.3, this means $a$ is neither orientation preserving nor orientation reversing and thus contradicts our restriction on $a$. Therefore, ga must equal $k$ for all $g$ between $j$ and $i$ and so by definition $(j, i) a=(k, k)$.

EXAMPLE 4.8. For orientation preserving or orientation reversing letters, Lemma 4.7 shows that for this diagram

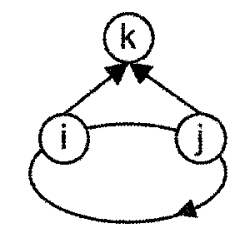

either everything in the segment from $i$ to $j$ goes to $k$ or everything in the segment from $j$ to $i$ does.

As in previous chapters, we can extend the definition of the transitions in the interval automaton to words $w \in A^{*}$. One can prove by induction that: 
- $\infty \cdot w=\infty$

- If $i w=j w=k$, then either $(i, j) \stackrel{w}{\longrightarrow}(k, k)$ or $(i, j) \stackrel{w}{\longrightarrow} \infty$.

- If $i w=k \neq j w=l$, then $(i, j) w= \begin{cases}(k, l), & \text { if } w \text { is orientation preserving; } \\ (l, k), & \text { if } w \text { is orientation reversing. }\end{cases}$ From these observations, we get the following result.

LEMMA 4.9. If $w \in A^{*}$ and $i, j \in Q$ such that $(i, j) w=(k, l) \neq \infty$ then

$$
[i, j] w \subseteq[k, l]
$$

Proof. Suppose $w$ is orientation preserving and $i w=k \neq l=j w$. If $m \in[i, j]$, we have that $i, m, j$ is in circular order. Hence $i w=k, m w, j w=l$ is in circular order. So $m w \in[k, l]$. Thus, $[i, j] w \subseteq[k, l]$. If $k=l$ and $m \in[i, j]$, then, since $(i, j) w \neq \infty$, we must have $m w=k$. Thus, $[i, j] w \subseteq[k, l]$. The orientation reversing case is similar.

EXAMPLE 4.10. For orientation preserving words, Lemma 4.9 is saying that for a word $w$ we have that either

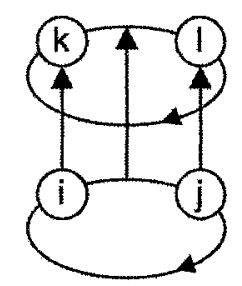

or

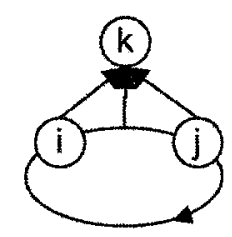

occurs, and there are no other alternatives if $(i, j) \neq \infty$

Given two intervals $(i, j)$ and $(k, l)$, we say that $(i, j)$ is a subinterval of $(k, l)$ if the sequence $k, i, j, l$ is in circular order. Notice with this definition that $(2,1)$ is not a subinterval of $(1, n)$ since $2,1, n, 1$ is not in circular order. 
LEMMA 4.11. If $w \in A^{*}$ and $(k, l) \in Q$ such that $(k, l) w \neq \infty$, then for any subinterval $(i, j)$ of $(k, l)$, we have that $(i, j) w \neq \infty$ and $(i, j) w$ is a subinterval of $(k, l) w$.

Proof. We use induction on the length of the word $w$. The base case when $w$ is empty is obvious. Let $w=v a$ for some word $v \in A^{*}$ and some letter $a \in A$. Since $(k, l) w \neq \infty$ and $\infty$ is a sink, $(k, l) v \neq \infty$. Thus, by induction, we get that $(i, j) v \neq \infty$ and is a subinterval of $(k, l) v$. It remains to show that $((i, j) v) a$ is a subinterval of $((k, l) v) a$. We shall denote $(i, j) v$ by $\left(i^{\prime}, j^{\prime}\right)$ and $(k, l) v$ by $\left(k^{\prime}, l^{\prime}\right)$. If $i^{\prime} a \neq j^{\prime} a$ or if $i^{\prime} a=j^{\prime} a=h a$ for all $h$ between $i^{\prime}$ and $j^{\prime}$, the result follows from the definition of the transition function. Suppose that $i^{\prime} a=j^{\prime} a=m$ with $h a \neq m$ for some $h$ between $i^{\prime}$ and $j^{\prime}$.

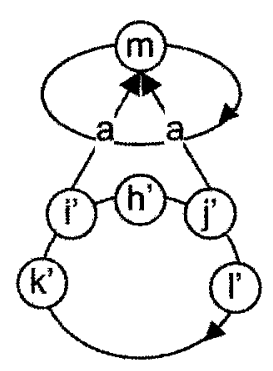

By Lemma 4.7, we have that $g a=m$ for all $g$ between $j^{\prime}$ and $i^{\prime}$. Since the sequence $k^{\prime}, i^{\prime}, j^{\prime}, l^{\prime}$ is in circular order, so is $j^{\prime}, l^{\prime}, k^{\prime}, i^{\prime}$. Therefore, $k^{\prime} a=l^{\prime} a=m$. Since $h a \neq m$ and $h$ is between $k^{\prime}$ and $l^{\prime}$, we get that $\left(k^{\prime}, l^{\prime}\right) a=\infty$ and this is clearly a contradiction. This completes the proof.

EXAMPLE 4.12. For orientation preserving words, Lemma 4.11 says for a word $w$ we have that either

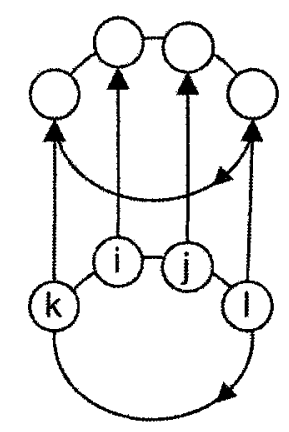


or

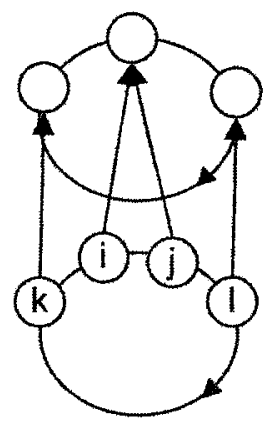

or

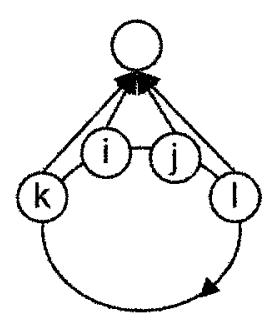

occurs, and there are no other alternatives if $(i, j) w \neq \infty$.

LEMma 4.13. Let $(Q, A)$ be a weakly orientable automaton, and let $w \in A^{*}$. Then for each $k \in\{1, \ldots, n\}$ there is an interval $(i, j)$ of $Q$ representing the segment $k w^{-1}$ and such that $(i, j) w=(k, k)$.

Proof. We proceed by induction on the length of $w$. If $|w|=0$, then $w=\varepsilon$, $k w^{-1}=\{k\}, k w^{-1}$ is represented by $(k, k)$ and $(k, k) \varepsilon=(k, k)$. Suppose $w=x v$ with $v \in A^{*}, x \in A$. Then $k w^{-1}=k v^{-1} x^{-1}$. By induction $k v^{-1}$ is represented by an interval $(g, h)$ such that $(g, h) v=(k, k)$.

Case 1: $[g, h] x^{-1} \neq\{1, \ldots, n\}$.

Lemma 4.5 implies that $[g, h] x^{-1}$ is a segment and since it is proper, it is represented by a unique interval $(i, j)$. We first show that $(i, j) x \neq \infty$. Indeed, suppose that $(i, j) x=\infty$. Then by Lemma $4.7,(j, i) x=(l, l)$ where $l=i \cdot x=j \cdot x$. So $q x=l$ for all $q \in[j, i]$. Then $[j, i]$ has image under $x$ equal to $\{l\} \subseteq[g, h]$. But, $[i, j]=[g, h] x^{-1}$. Thus, $x$ takes $[i, j] \cup[j, i]$ into $[g, h]$. Hence, $[g, h] x^{-1} \supseteq[i, j] \cup[j, i]=\{1, \ldots, n\}$. This contradicts $[g, h] x^{-1} \neq\{1, \ldots, n\}$. Therefore, $(i, j) \cdot x \neq \infty$. 
By Lemma 4.11, we may now conclude that $(i, j) x$ is a subinterval of $(g, h)$. Again, by Lemma 4.11 , we get that $(i, j) w=(i, j) x v$ is a subinterval of $(g, h) v=(k, k)$. In this case, we are done since $(k, k)$ is the only subinterval of $(k, k)$.

Case 2: $[g, h] x^{-1}=\{1, \ldots, n\}$.

Choose $(p, q)$ with the size of $[p, q]$ smallest amongst all subintervals of $(g, h)$ such that $[p, q] x^{-1}=\{1, \ldots, n\}$. Observe that $p, q$ are in $\{1, \ldots, n\} \cdot x$. Otherwise, we can find a smaller interval by moving right from $p$ and left from $q$ to the first element that belongs to the image of $x$. If $p=q$, then $\{1, \ldots, n\} x=\{p\}$ and so $(i, j) x=(p, p)$ for any interval $(i, j)$ representing $\{1, \ldots, n\}$. Thus,

$$
(i, j) w=(i, j) x v=(p, p) v=(k, k)
$$

since $p \in[g, h]=k v^{-1}$.

Now suppose that $p \neq q$.

Subcase 1: $x$ is orientation preserving.

Then $q x^{-1}$ is a proper subset of $\{1, \ldots, n\}$ since $p \neq q$. By Lemma $4.5, q x^{-1}$ is a proper segment, and so it has a unique representation as an interval $(m, j)$. Choose $i$ such that $j$ immediately precedes $i$. Then $i x \in[p, q]$ and $i x \neq q$ since $i$ is immediately after $j$. Suppose that $i x \neq p$ as well. Since $p \in\{1, \ldots, n\} \cdot x$, there exists $r \in[i, m]$ such that $r x=p$.

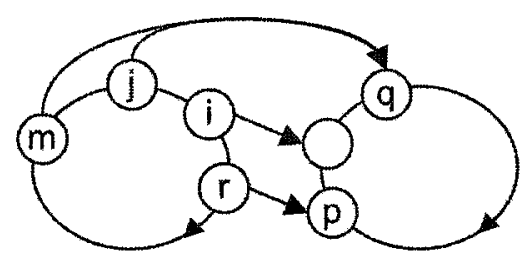

Notice that $j, i, r$ are in circular order. Thus, $j x, i x, r x$ should be in circular order since $x$ is orientation preserving. But, in the diagram, we see that $q=j x, p=r x, i x$ are in circular order. This is a contradiction. Therefore, $i x=p$ and $(i, j) x=(p, q)$.

Subcase 2: $x$ is orientation reversing. 
Then $p x^{-1}$ is a proper segment of $\{1, \ldots, n\}$ and it can be represented by a unique interval $(m, j)$. As before, choose $i$ such that $j$ immediately precedes $i$. Again, $i x \in$ $[p, q]$. Also, $i x \neq p$ since $i$ is immediately after $j$. Suppose that $i x \neq q$ as well. Since $q \in\{1, \ldots, n\} \cdot x$, there exists $r \in[i, m]$ such that $r x=q$.

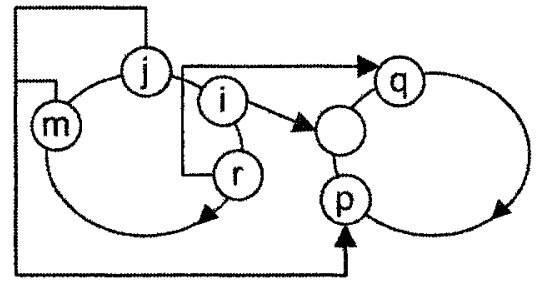

As before, $j, i, r$ are in circular order. Since $x$ is orientation reversing, $r x, i x, j x$ should be in circular order. But, in the diagram, we see that $q=r x, p=j x, i x$ is in circular order. This is a contradiction. Therefore, $i x=q$ and $(i, j) x=(p, q)$.

In both of these subcases, we have that $(i, j) x=(p, q)$. Thus,

$$
(i, j) w=(i, j) x v=(p, q) v
$$

which is a subinterval of $(g, h) v=(k, k)$ by Lemma 4.11. Thus, $(i, j) w=(k, k)$ as required.

Theorem 4.14 (Ananichev, Volkov [1]). For a weakly orientable automaton $(Q, A)$, $w \in A^{*}$ is a synchronizing word if and only if there exists $(i, j)$ representing all of $Q$ such that $(i, j) w=(k, k)$ for some $k \in Q$. Hence there is a synchronizing word of length at most $(n-1)^{2}$.

ProOF. First we show that $w$ is a synchronizing word if and only if $w$ sends some interval representing the whole set to an interval representing a single state.

Let $w$ be a synchronizing word. Then there exists $k \in\{1, \ldots, n\}$ such that $i w=k$ for all $i \in\{1, \ldots, n\}$. By Lemma $4.13, k w^{-1}$ is represented by an interval $(i, j)$ such that $[i, j]=\{1, \ldots, n\}$ and $(i, j) w=(k, k)$. Conversely, if $(i, j) w=(k, k)$ where $[i, j]=\{1, \ldots, n\}$, then by Lemma $4.9,[i, j] w \subseteq[k, k]$ and so $w$ is synchronizing to $k$. Thus, we have established that $w$ is synchronizing if and only if $(i, j) w=(k, k)$ for some interval $(i, j)$ representing $\{1, \ldots, n\}$. 
Any minimal synchronizing word $w$, when run in the interval automaton, avoids the sink state $\infty$, should not visit any state twice, should only visit exactly one interval representing $\{1, \ldots, n\}$ (otherwise we could just start from the last such interval seen to get a smaller word), and should only visit exactly one interval of the form $(k, k)$ (since we can stop once we reach such a state). Therefore, we visit at most a total of

$$
\left(n^{2}+1\right)-1-(n-1)-(n-1)=n^{2}-2 n+2
$$

states. But, since a path of length $k$ visits $k+1$ states, a minimal synchronizing word is of length at most $n^{2}-2 n+1=(n-1)^{2}$.

Eppstein proved the special case where $(Q, A)$ has just orientation preserving maps $[7]$. 
CHAPTER 5

\section{Linearization of Automata}

In this chapter we will consider how to express an automaton $(Q, A)$ using concepts from linear algebra over the vector space $\mathbb{Q}^{n}$ where $n=|Q|$. For any $a \in A$, we define $M_{a}$ to be the incidence matrix of the graph consisting of only the edges labelled by $a$. That is,

$$
\left(M_{a}\right)_{i j}= \begin{cases}1, & \text { if } i \stackrel{a}{\longrightarrow} j ; \\ 0, & \text { else. }\end{cases}
$$

Notice that if we add up all of these $M_{a}$ for $(Q, A)$, we obtain the incidence matrix for the underlying unlabelled graph since the labels partition the edges.

EXAMPLE 5.1. Recall the Černý example from Theorem 2.7. Then, for the case when $n=4$, we will have the following matrices for the two letter input $\{a, b\}$ :

$$
M_{a}=\left(\begin{array}{llll}
0 & 1 & 0 & 0 \\
0 & 0 & 1 & 0 \\
0 & 0 & 0 & 1 \\
1 & 0 & 0 & 0
\end{array}\right)
$$

and

$$
M_{b}=\left(\begin{array}{llll}
1 & 0 & 0 & 0 \\
0 & 0 & 1 & 0 \\
0 & 0 & 1 & 0 \\
0 & 0 & 0 & 1
\end{array}\right) .
$$

Notice that the matrix $M_{a}$ corresponds to the permutation

$$
\left(\begin{array}{llll}
1 & 2 & 3 & 4 \\
2 & 3 & 4 & 1
\end{array}\right) .
$$

27 
But, $M_{b}$ does not correspond to a permutation.

Also,

$$
M_{a}+M_{b}=\left(\begin{array}{cccc}
1 & 1 & 0 & 0 \\
0 & 0 & 2 & 0 \\
0 & 0 & 1 & 1 \\
1 & 0 & 0 & 1
\end{array}\right)
$$

is the incidence matrix for the unlabelled graph.

For words $w=a_{1} \ldots a_{n} \in A^{*}$, we define $M_{w}=M_{a_{1}} \ldots M_{a_{n}}$. One has that,

$$
\left(M_{w}\right)_{i j}= \begin{cases}1, & \text { if } i \stackrel{w}{\longrightarrow} j ; \\ 0, & \text { else }\end{cases}
$$

and since we are allowed to have an empty string, we define $M_{\varepsilon}=I$, the $n \times n$ identity matrix. Observe that

$$
\left(M_{w}^{t}\right)_{x y}= \begin{cases}1, & \text { if } y \stackrel{w}{\longrightarrow} x \\ 0, & \text { else. }\end{cases}
$$

So it is reasonable to define $M_{w^{-1}}=M_{w}^{t}$.

We shall also associate a row vector $[S]$ to $S \subseteq Q$ such that,

$$
[S]_{i}= \begin{cases}1, & \text { if } i \in S \\ 0, & \text { else. }\end{cases}
$$

In particular, $[Q]=[1, \ldots, 1]$.

EXAMPLE 5.2. If $Q=\{1,2,3,4\}$ and $S=\{2,3\}$, then $[S]=[0,1,1,0]$.

We can describe how the automaton functions in terms of our matrices. For example,

$$
[\{i\}] M_{w}=[\{i \cdot w\}]
$$

Also, we have the following proposition. 
Proposition 5.3. If $S \subseteq Q$ and $w \in A^{*}$, then $\left[S w^{-1}\right]=[S] M_{w^{-1}}=[S] M_{w}^{t}$.

ProOF.

$$
\begin{aligned}
\left([S] M_{w^{-1}}\right)_{i} & =\left([S] M_{w}^{t}\right)_{i} \\
& =\sum_{k=1}^{n}[S]_{k}\left(M_{w}^{t}\right)_{k i} \\
& =\sum_{k=1}^{n}[S]_{k}\left(M_{w}\right)_{i k} \\
& =[S]_{i \cdot w}
\end{aligned}
$$

since

$$
\left(M_{w}\right)_{i k}= \begin{cases}1, & \text { if } i \stackrel{w}{\longrightarrow} k \\ 0, & \text { else. }\end{cases}
$$

Hence,

$$
\begin{aligned}
\left([S] M_{w^{-1}}\right)_{i} & = \begin{cases}1, & \text { if } i \cdot w \in S \\
0, & \text { else. }\end{cases} \\
& = \begin{cases}1, & \text { if } i \in S w^{-1} \\
0, & \text { else. }\end{cases}
\end{aligned}
$$

Thus, $[S] M_{w^{-1}}=\left[S w^{-1}\right]$.

Recall that our strategy for obtaining a synchronizing word is to find for any nonempty proper subset $S \subseteq Q$ a word $u \in A^{*}$ such that $\left|S u^{-1}\right|>|S|$. We can use the above to reformulate $|S|$ as

$$
|S|=\sum_{i=1}^{n}[S]_{i}=\langle[S],[Q]\rangle
$$

Thus,

$$
\left|S u^{-1}\right|=\left\langle[S] M_{u^{-1}},[Q]\right\rangle=\left\langle[S] M_{u}^{t},[Q]\right\rangle=\left\langle[S],[Q] M_{u}\right\rangle
$$


DEFINITION 5.4. Define, for a word $w \in A^{*}$ and a subset $S \subseteq Q, \alpha_{S}(w)=$ $\left|S w^{-1}\right|-|S|$. We aim to compute $\alpha_{S}(w)$.

LEMMA 5.5. $[Q]\left(M_{w}-I\right) \perp[Q]$.

Proof. To prove this lemma, we must show that $\left\langle[Q],[Q]\left(M_{w}-I\right)\right\rangle=0$. Indeed,

$$
\left\langle[Q],[Q]\left(M_{w}-I\right)\right\rangle=\left\langle[Q] M_{w}^{t},[Q]\right\rangle-\langle[Q],[Q]\rangle
$$

But, $[Q] M_{w}^{t}=\left[Q w^{-1}\right]=[Q]$. Therefore, the right hand side of (5.1) is equal to zero.

The following subspace of $\mathbb{Q}^{n}$ will play an important role in this thesis. Let

$$
V_{0}=\left\{v=\left[c_{1}, \ldots, c_{n}\right] \in \mathbb{Q} \mid c_{1}+\ldots+c_{n}=0\right\}=[Q]^{\perp}
$$

Proposition 5.6. Let $w \in A^{*}$ and $S \subseteq Q$. Also, let $[S]=S^{\prime}+U$, where $S^{\prime} \in[Q]^{\perp}$ and $U \in \operatorname{span}\{[Q]\}$, be the orthogonal decomposition. Then,

$$
\begin{aligned}
\alpha_{S}(w) & =\left\langle S^{\prime} M_{w}^{t},[Q]\right\rangle \\
& =\left\langle S^{\prime},[Q] M_{w}\right\rangle \\
& =\left\langle S^{\prime},[Q]\left(M_{w}-I\right)\right\rangle \\
& =\left\langle S^{\prime}\left(M_{w}^{t}-I\right),[Q]\right\rangle
\end{aligned}
$$

Proof. We shall try to manipulate the definition of $\alpha_{S}(w)$ in order to obtain the desired result.

$$
\begin{aligned}
\alpha_{S}(w) & =\left|S w^{-1}\right|-|S| \\
& =\left\langle[S] M_{w}^{t},[Q]\right\rangle-\langle[S],[Q]\rangle \\
& =\left\langle[S]\left(M_{w}^{t}-I\right),[Q]\right\rangle
\end{aligned}
$$




$$
\begin{aligned}
& =\left\langle[S],[Q]\left(M_{w}-I\right)\right\rangle \\
& =\left\langle S^{\prime}+U,[Q]\left(M_{w}-I\right)\right\rangle \\
& =\left\langle S^{\prime},[Q]\left(M_{w}-I\right)\right\rangle+\left\langle U,[Q]\left(M_{w}-I\right)\right\rangle \\
& =\left\langle S^{\prime},[Q]\left(M_{w}-I\right)\right\rangle
\end{aligned}
$$

by Lemma 5.5 since $U \in \operatorname{span}\{[Q]\}$.

Thus we have shown that $\alpha_{S}(w)=\left\langle S^{\prime},[Q]\left(M_{w}-I\right)\right\rangle$. Since $S^{\prime} \in[Q]^{\perp}$, we may finish the proof as follows:

$$
\begin{aligned}
\alpha_{S}(w) & =\left\langle S^{\prime},[Q]\left(M_{w}-I\right)\right\rangle \\
& =\left\langle S^{\prime},[Q] M_{w}\right\rangle-\left\langle S^{\prime},[Q]\right\rangle \\
& =\left\langle S^{\prime},[Q] M_{w}\right\rangle
\end{aligned}
$$

This completes the proof.

Proposition 5.7. If $v \in V_{0}$, then $v \cdot M_{w} \in V_{0}$ for all $w \in A^{*}$.

Proof. Let $v_{0} \in V_{0}$. Then,

$$
\begin{aligned}
\left\langle v_{0} M_{w},[Q]\right\rangle & =\left\langle v_{0},[Q] M_{w}^{t}\right\rangle \\
& =\left\langle v_{0},\left[Q w^{-1}\right]\right\rangle \\
& =\left\langle v_{0},[Q]\right\rangle=0
\end{aligned}
$$

So, $v_{0} M_{w} \in[Q]^{\perp}=V_{0}$.

Clearly $\left\{e_{1}-e_{2}, \ldots, e_{1}-e_{n}\right\}$ is a basis of $V_{0}$. Thus, $\operatorname{dim}\left(V_{0}\right)=n-1$. Define $e_{i j}=e_{i}-e_{j}$. With this notation $V_{0}$ has basis $\left\{e_{12}, \ldots, e_{1 n}\right\}$. Notice that $e_{i j} M_{w}=$ $e_{i} M_{w}-e_{j} M_{w}=e_{i \cdot w}-e_{j \cdot w}$. Thus, $w$ collapses $\{i, j\}$ if and only if $e_{i j} M_{w}=0$. Therefore, $w$ is a synchronizing word if and only if $e_{1 i} M_{w}=0$ for $2 \leq i \leq n$. In other words, $w$ 
is a synchronizing word if and only if $M_{w}: V_{0} \rightarrow V_{0}$ is the zero transformation. This is essentially a reformulation of Lemma 3.2 .

Definition 5.8. A matrix $M$ is a 0-1 row monomial if each row contains only a single entry that is 1 and the rest are 0 .

Notice that $M_{w}$, for all $w \in A^{*}$, is row monomial. With this we can now rephrase Črný's Conjecture.

Rephrasing Černý's Conjecture: If $M_{a_{1}}, \ldots, M_{a_{m}}$ are $n \times n$ 0-1 row monomial matrices such that there exists a product of $M_{a_{i}}$ which is the zero transformation on $V_{0}$, then this product can be chosen to have length at most $(n-1)^{2}$.

A related problem is the Mortality Problem, where we have arbitrary $n \times n$ matrices $A_{1}, \ldots, A_{k}$ over $\mathbb{Q}$ and we try to determine if the zero matrix can be expressed as a product of these matrices. This problem is undecidable [14]. Our problem is clearly decidable, though, since we need only try all words within the cubic upper bound on the length of a synchronizing word.

The following lemma from [8] is useful when dealing with the representation of free monoids; see [4] for a similar lemma.

LEMMA 5.9. Let $U$ be a subspace of $\mathbb{Q}^{n}$ and $0 \neq x \in U$. Let $A$ be a finite alphabet and suppose we have for all $a \in A$ a linear transformation $M_{a}: \mathbb{Q}^{n} \rightarrow \mathbb{Q}^{n}$.

Define $M_{\varepsilon}=I$ and for any word $w=a_{1} a_{2} \ldots a_{n} \in A^{*}$, define $M_{w}=M_{a_{1}} M_{a_{2}} \ldots M_{a_{n}}$. Then if there exists $w \in A^{*}$ such that $x M_{w} \notin U$, we can choose $w$ to have length at most $\operatorname{dim}(U)$.

PROOF. For this proof we shall consider vector subspaces of the form

$$
U_{i}=\operatorname{span}\left\{x M_{w}|| w \mid \leq i\right\}
$$

Since there are no words of length zero except the empty word, $U_{0}=\operatorname{span}\{x\}$. Then $\operatorname{dim}\left(U_{0}\right)=\operatorname{dim}(\operatorname{span}\{x\})=1$. Also, 


$$
U_{0} \subseteq U_{1} \subseteq \ldots
$$

Hence, since $\operatorname{dim}\left(U_{i}\right) \leq \operatorname{dim}\left(U_{i+1}\right)$, this increasing sequence of subspaces can not consist entirely of distinct subspaces. Now we suppose that for some $i$ we have $U_{i}=$ $U_{i+1}$.

CLAIM 5.10. If $U_{i}=U_{i+1}$, then $U_{i}=U_{i+j}$ for all $j \geq 1$.

Proof. Let $a \in A$ and $w \in A^{*}$, where $|w| \leq i$. Then,

$$
x M_{w} M_{a}=x M_{w a} \in U_{i+1}=U_{i}
$$

Thus, $U_{i} M_{a} \subseteq U_{i}$. But, $U_{i} M_{a} \subseteq U_{i}$ for all $a \in A$ implies that $U_{i} M_{v} \subseteq U_{i}$ for all $v \in A^{*}$. Thus, for all $w \in A^{*}, x M_{w} \in U_{i}$. Hence $U_{i+j} \subseteq U_{i}$ for all $j>0$ by definition.

Now suppose there is a $w \in A^{*}$ such that $x M_{w} \notin U$, and we take such a $w$ of minimal length. If $|w|=i$, then $U_{i} \nsubseteq U$ since $x M_{w} \in U_{i}$. Since $w$ is the shortest possible, $U_{i-1} \subseteq U$ by the definition of $U_{i-1}$. This means that $U_{i-1} \neq U_{i}$. Furthermore, we can not have $U_{j}=U_{j+1}$ for $j+1 \leq i-1$ by the claim. Thus, $U_{0} \subset U_{1} \subset \ldots \subset U_{i-1}$ and as a consequence $1=\operatorname{dim}\left(U_{0}\right)<\operatorname{dim}\left(U_{1}\right)<\ldots<\operatorname{dim}\left(U_{i-1}\right)$. Therefore, $|w|=i \leq$ $\operatorname{dim}\left(U_{i-1}\right) \leq \operatorname{dim}(U)$

We will now introduce other concepts from linear algebra that will be useful later on.

DEFINITION 5.11. If $B$ is an $n \times n$ square matrix, we call the determinant of $B-\lambda I$ the characteristic polynomial of $B$, denoted by $f_{B}(\lambda)$.

DEFINITION 5.12. The minimal polynomial of a square matrix $B$ is the monic polynomial $g_{B}(\lambda)$ of minimal degree such that $g_{B}(B)=0$. (Similarly for linear transformations.)

If $f$ is a polynomial with $f(B)=0$, then $g_{B}(x)$ divides $f(x)$. 
DeFINITION 5.13. Let $F[x]$ denote the ring of polynomials over a field $F$. $A$ non-constant polynomial $f(x) \in F[x]$ is called irreducible if $f(x)=g(x) h(x)$ with $g(x), h(x) \in F[x]$ implies $g(x)$ or $h(x)$ is constant.

DEFINITION 5.14. If $G$ is a group of matrices acting on a vector space $V$, a subspace $W \subseteq V$ is called $G$-invariant if $W G \subseteq W$.

DEFINITION 5.15. $W$ is $G$-irreducible if the only $G$-invariant subspaces of $W$ are $\{0\}, W$.

The reader is referred to $[\mathbf{1 0}]$ for more on these notions. 


\section{CHAPTER 6}

\section{Eulerian Automata}

In this chapter we shall use concepts from Chapter 5 to consider Eulerian automata. A directed graph is said to be Eulerian if the in-degree is the same as the out-degree for each vertex and any two vertices can be connected by a directed path. We call an automaton $(Q, A)$ Eulerian if the underlying graph is Eulerian. Since the out-degree of each vertex is $|A|=k$, the in-degree is $k$ as well. Kari proved [8] that for Eulerian automata, one can do better than $(n-1)^{2}$ for the length of the minimal synchronizing word.

Theorem 6.1 (Kari $[8])$. Let $(Q, A)$ be an Eulerian automaton. If $(Q, A)$ is synchronizing, then there exists a synchronizing word of length at most

$$
(n-2)(n-1)+1=n^{2}-3 n+3 .
$$

Proof. Recall from Chapter 5 that we defined $\alpha_{S}(w)=\left|S w^{-1}\right|-|S|$. According to our strategy from Chapter 2, it suffices to show that for each proper non-empty subset $S$ of $Q$, we can find a word $w$ of length at most $n-1$ such that $\alpha_{S}(w)>0$.

Let $[S]=S^{\prime}+U$ be the orthogonal decomposition with $S^{\prime} \in V_{0}$ and $U \in \operatorname{span}\{[Q]\}$ as in Proposition 5.6. Since $\emptyset \neq S \subset Q,[S] \notin \operatorname{span}\{[Q]\}$, and so we have $S^{\prime} \neq 0$. Since $(Q, A)$ is synchronizing and Eulerian, by Proposition 2.4 there exists a synchronizing word $w$ into $S$. Thus, $\alpha_{S}(w)>0$. By Proposition 5.6, for any $v \in A^{*}, \alpha_{S}(v)=$ $\left\langle S^{\prime},[Q] M_{v}\right\rangle$. So, in particular,

$$
\alpha_{S}(w)=\left\langle S^{\prime},[Q] M_{w}\right\rangle>0
$$

Thus, $[Q] M_{w} \notin\left(S^{\prime}\right)^{\perp}$. By Lemma 5.9, there exists $v \in A^{*}$ such that $|v| \leq \operatorname{dim}\left(\left(S^{\prime}\right)^{\perp}\right)=$ $n-1$ and $[Q] M_{v} \notin\left(S^{\prime}\right)^{\perp}$. 
Thus,

$$
0 \neq\left\langle S^{\prime},[Q] M_{v}\right\rangle=\alpha_{S}(v)
$$

We would like a new word $u$ of length at most $n-1$ such that $\alpha_{S}(u)>0$.

Let $B$ be the incidence matrix of the underlying unlabelled graph. Then we saw that $B=\sum_{a \in A} M_{a}$. More generally, since $\left(B^{r}\right)_{i j}$ is the number of paths of length $r$ from $i$ to $j$ in the underlying graph, we see by partitioning paths by their labels that

$$
B^{r}=\sum_{|w|=r} M_{w}
$$

where $w \in A^{*}$. Since the graph is Eulerian, $[Q] B=k[Q]$, where $k=|A|$ is the indegree, since position $i$ of $[Q] B$ is the sum of the entries of column $i$. Thus, $[Q] B^{r}=$ $k^{r}[Q]$. So,

$$
\begin{aligned}
0 & =[Q]\left(B^{r}-k^{r} I\right) \\
& =[Q]\left(\sum_{|w|=r} M_{w}-k^{r} I\right) \\
& =\sum_{|w|=r}[Q]\left(M_{w}-I\right)
\end{aligned}
$$

since there are $k^{r}$ words of length $r$. Therefore,

$$
\begin{aligned}
0 & =\left\langle U, \sum_{|w|=r}[Q]\left(M_{w}-I\right)\right\rangle \\
& =\sum_{|w|=r}\left\langle U,[Q]\left(M_{w}-I\right)\right\rangle \\
& =\sum_{|w|=r} \alpha_{S}(w)
\end{aligned}
$$

by Proposition 5.6.

Since there exists $0 \leq r \leq n-1$ such that $\alpha_{S}(v) \neq 0$ for some $v \in A^{*}$ with $|v|=r$, there must be a word $w$ of length $r$ such that $\alpha_{S}(w)>0$ in order for (6.3) to be zero. This completes the proof. 
EXAMPLE 6.2. We construct an Eulerian automaton as follows:

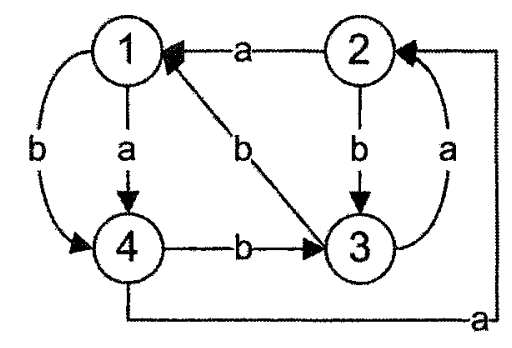

For this example, a synchronizing word is bab and leaves the automaton in state 3 . This is clearly the shortest since we need at least three letters to expand the set containing only state 3 to the set containing all states. 


\section{CHAPTER 7}

\section{Irreducible Permutation Automata}

In this chapter, we will use concepts from Chapter 5 to consider circular automata $(Q, A)$ with $Q=\{1, \ldots, q\}$ where $q$ is a prime number. Let $p$ be a letter acting as a cyclic permutation on $Q$. Our goal is to prove Pin's theorem $[15]$ that $(Q, A)$ is synchronizing if $A$ contains a non-permutation and that a minimal synchronizing word for $(Q, A)$ has length at most $(q-1)^{2}$. Our proof (unpublished) is due to $\mathrm{B}$. Steinberg and establishes a more general result. Consider the vector space $V$ over $\mathbb{Q}^{q}$ with basis $\left\{e_{1}, \ldots, e_{q}\right\}$ as we had in Chapter 5 . Let $G=\langle p\rangle$ be the group generated by $p$. When $g \in G$, we shall sometimes write $M_{g}$ for $M_{w}$ where $w \in\{p\}^{*}$ represents g. Recall that

$$
V_{0}=[Q]^{\perp}=\left\{v=\left[c_{1}, \ldots, c_{q}\right] \mid \sum_{i=1}^{q} c_{i}=0\right\} .
$$

Also, recall from Proposition 5.7 that if $v \in V_{0}$, then $v M_{w} \in V_{0}$ for all $w \in A^{*}$. In particular, $V_{0}$ is $G$-invariant.

Proposition 7.1. $V_{0}$ is G-irreducible.

Proof. Without loss of generality, we may assume, by renumbering, that

$$
p=\left(\begin{array}{cccc}
1 & 2 & \ldots & q \\
2 & 3 & \ldots & 1
\end{array}\right)
$$

In this case, $G=\langle p\rangle \cong \mathbb{Z}_{q}$. Then $M_{p}$ is the $q \times q$ permutation matrix that encodes this permutation. Using the cofactor expansion on the first column of $M_{p}-\lambda I$, we calculate the characteristic polynomial as follows: 


$$
\begin{aligned}
f_{p}(\lambda) & =\operatorname{det}\left(\begin{array}{ccccc}
-\lambda & 1 & 0 & \ldots & 0 \\
0 & -\lambda & 1 & \ddots & \vdots \\
\vdots & 0 & \ddots & \ddots & 0 \\
0 & \vdots & \ddots & -\lambda & 1 \\
1 & 0 & \ldots & 0 & -\lambda
\end{array}\right) \\
& =(-\lambda) \operatorname{det}\left(\begin{array}{cccc}
-\lambda & 1 & 0 & \ldots \\
0 & \ddots & \ddots & \ddots \\
\vdots & \ddots & -\lambda & 1 \\
0 & \ldots & 0 & -\lambda
\end{array}\right)+(-1)^{q+1} \operatorname{det}\left(\begin{array}{cccc}
1 & 0 & \ldots & 0 \\
-\lambda & \ddots & \ddots & \vdots \\
0 & \ddots & 1 & 0 \\
\vdots & \ddots & -\lambda & 1
\end{array}\right) \\
& =-\lambda(-\lambda)^{q-1}+(-1)^{q+1} \\
& =(-1)^{q}\left(\lambda^{q}-1\right) \\
& =(-1)^{q}(\lambda-1)\left(1+\lambda+\ldots+\lambda^{q-1}\right) .
\end{aligned}
$$

We recall that $1+\lambda+\ldots+\lambda^{q-1}$ is irreducible over $\mathbb{Q}[\mathbf{1 3}]$.

Now we consider $M_{p}$ as a linear transformation $M_{p}: V_{0} \rightarrow V_{0}$. Recall that $\operatorname{dim}\left(V_{0}\right)=q-1$. By the Cayley-Hamilton Theorem, $f_{p}\left(M_{p}\right)=0$ on $V$ and hence on $V_{0}$. Thus, the minimal polynomial $g_{p}(\lambda)$ for $M_{p}$ on $V_{0}$ divides $f_{p}(\lambda)$. Then we have the following choices for $g_{p}(\lambda): f_{p}(\lambda), \lambda-1$, and $1+\lambda+\ldots+\lambda^{q-1}$. But, $f_{p}(\lambda)$ is not possible since

$$
\operatorname{deg}\left(f_{p}(\lambda)\right)>\operatorname{dim}\left(V_{0}\right) \geq \operatorname{deg}\left(g_{p}(\lambda)\right)
$$

Also, $\lambda-1$ is not possible since $M_{p}-I=0$ implies that $M_{p}=I$ on $V_{0}$. The next claim shows this can't happen.

CLAIM 7.2. If $v \in V_{0}$ and $v M_{p}=v$, then $v=0$.

Proof. Let $v=\left[c_{1}, \ldots, c_{q}\right]$. Then,

$$
\left[c_{1}, c_{2}, \ldots, c_{q}\right] M_{p}=\left[c_{q}, c_{1}, \ldots, c_{q-1}\right]
$$


Hence, if $v M_{p}=v$, then $c_{q}=c_{1}, c_{1}=c_{2}, \ldots, c_{q-1}=c_{q}$ and so $c_{1}=\ldots=c_{q}$. Since $v \in V_{0}$, then

$$
0=\sum_{i=1}^{q} c_{i}=q c_{1} \Rightarrow c_{i}=0 \text { for all } i
$$

Thus, $v$ is equal to zero.

In particular, $M_{p} \neq I$ on $V_{0}$. Therefore, the minimal polynomial $g_{p}(\lambda)$ must be $1+\lambda+\ldots+\lambda^{q-1}$.

Suppose that $\{0\} \neq W \subseteq V_{0}$ is a subspace such that $W M_{p} \subseteq W$, i.e. $W$ is $G$ invariant. We can then view $M_{p}: W \rightarrow W$ as a linear transformation. By restriction, $g_{p}\left(M_{p}\right)=0$ on $V_{0}$ and hence on $W$. Thus, the minimal polynomial $h_{p}(\lambda)$ of $M_{p}$ on $W$ divides $g_{p}(\lambda)$. Since $g_{p}(\lambda)$ is irreducible over $\mathbb{Q}$, either $h_{p}(\lambda)=1$ or $h_{p}(\lambda)=g_{p}(\lambda)$. But the minimal polynomial is never a constant, therefore $g_{p}(\lambda)$ is the minimal polynomial. We now claim that $\operatorname{dim}(W)=q-1$. Indeed, the degree of the minimal polynomial is at most the degree of the characteristic polynomial which equals the dimension of $W$. Thus,

$$
q-1 \leq \operatorname{dim}(W) \leq \operatorname{dim}\left(V_{0}\right)=q-1
$$

So, $\operatorname{dim}(W)=q-1$. Thus, $W=V_{0}$, and therefore $V_{0}$ is $G$-irreducible.

Let us consider the more general situation, where $(Q, A)$ is an automaton with $Q=$ $\{1, \ldots, n\}$ and $P \subseteq A$ is a set of permutations containing a non-trivial permutation such that $V_{0}$ is $G$-irreducible where $G=\langle P\rangle$. Moreover, we assume $A$ has at least one non-permutation. Also, we can assume that $n>2$ since any non-permutation letter is a synchronizing for a two state automaton.

For example, $G$ is called 2-transitive [10] if for all ordered pairs $(i, j),(k, l)$ of elements of $Q$, there is a word $w \in P^{*}$ such that $i w=k$ and $j w=l$. It is known that in this situation $V_{0}$ is $G$-irreducible [10]. For instance, if

$$
P=\{(12),(23), \ldots,(n-1 n)\}
$$

then $G=\langle P\rangle=S_{n}$, which is the group of all permutations. This is 2-transitive [10]. 
We're going to show that $(Q, A)$ is synchronizing and estimate the length of a synchronizing word. Recall from Definition 5.4 that $\alpha_{S}(w)=\left|S w^{-1}\right|-|S|$. Also, if $g \in G, u \in A^{*}$, then we shall interpret $\alpha_{S}(u g)$ as $\left|S g^{-1} u^{-1}\right|-|S|$. Let $[S]=S^{\prime}+U$ be the orthogonal decomposition with $S^{\prime} \in V_{0}$, and $U \in \operatorname{span}\{[Q]\}$ as in Proposition 5.6. Since $\emptyset \neq S \subset Q,[S] \notin \operatorname{span}\{[Q]\}$, and so we have $S^{\prime} \neq 0$.

LEMma 7.3. Given a non-empty $S \subseteq Q$. Suppose $a \in A$ is any non-permutation, then there exists $g \in G$ such that $\alpha_{S}(a g) \neq 0$.

ProOF. There is nothing to prove when $\alpha_{S}(a) \neq 0$ since we can simply take $g=1$. Hence, it suffices to consider the case when $\alpha_{S}(a)=0$ since

$$
0=\alpha_{S}(a)=\left\langle S^{\prime},[Q]\left(M_{a}-I\right)\right\rangle
$$

Thus, $S^{\prime} \perp[Q]\left(M_{a}-I\right)$.

Claim 7.4. $[Q]\left(M_{a}-I\right) \neq 0$.

Proof. If $[Q]\left(M_{a}-I\right)=0$, then $[Q] M_{a}=[Q]$ and hence, $a$ is a permutation. But, this contradicts our choice of $a$.

Let $W=\operatorname{span}\left\{[Q]\left(M_{a}-I\right) M_{g} \mid g \in G\right\}$. Note that $W \neq\{0\}$ since $[Q]\left(M_{a}-I\right) \in$ $W$.

Proposition 7.5. $W$ is G-invariant.

Proof. Since $[Q]\left(M_{a}-I\right) \in V_{0}$ by Lemma 5.5 and for all $x \in A, V_{0} M_{x} \subseteq V_{0}$ by Proposition 5.7, we have $W \subseteq V_{0}$. Now if $p \in P$,

$$
[Q]\left(M_{a}-I\right) M_{g} M_{p}=[Q]\left(M_{a}-I\right) M_{g p} \in W
$$

since $g p \in G$. Therefore, since $G=\langle P\rangle, W$ is $G$-invariant.

Hence, by $G$-irreducibility, $W=V_{0}$. We know that $S^{\prime} \in V_{0}$, and this implies that $S^{\prime} \in W$. Since $0 \neq S^{\prime}$, we have that $S^{\prime} \notin W^{\perp}$. Since $W$ is spanned by $[Q]\left(M_{a}-I\right) M_{g}$, 
$g \in G$, there exists $g \in G$ such that

$$
\begin{aligned}
0 & \neq\left\langle S^{\prime},[Q]\left(M_{a}-I\right) M_{g}\right\rangle \\
& =\left\langle S^{\prime},[Q] M_{a} M_{g}\right\rangle-\left\langle S^{\prime},[Q] M_{g}\right\rangle \\
& =\alpha_{S}(a g)-\left\langle S^{\prime},[Q]\right\rangle \\
& =\alpha_{S}(a g)
\end{aligned}
$$

where the passage from (7.2) to (7.3) follows by Proposition 5.6 and since $M_{g}$ is a permutation matrix, while the last equality follows since $S^{\prime} \perp[Q]$.

LEMMa 7.6. Suppose $v \in V_{0}$. Then $v\left(\sum_{h \in G} M_{h}\right)=0$.

Proof. Clearly if $v=0$, then the statement holds. Hence is suffices to consider the case when $v \neq 0$, and we let $U=\operatorname{span}\left\{v \cdot\left(\sum_{h \in G} M_{h}\right)\right\}$.

We claim that $U$ is $G$-invariant. Indeed, let $p \in P$. Then

$$
\left(v \cdot\left(\sum_{h \in G} M_{h}\right)\right) \cdot M_{p}=v \cdot\left(\sum_{h \in G} M_{h p}\right)
$$

Since $p \in P$ is invertible, we can change subscripts to $h^{\prime}=h p$. So the right hand side of $(7.5)$ is $v \cdot\left(\sum_{h^{\prime} \in G} M_{h^{\prime}}\right) \in U$. Thus, $U$ is $G$-invariant.

Thus, we know that either $U=\{0\}$ or $V_{0}$ by $G$-irreducibility for $V_{0}$. Since $U=$ $\operatorname{span}\left\{v\left(\sum_{h \in G} M_{h}\right)\right.$, we have that $\operatorname{dim}(U) \leq 1<\operatorname{dim}\left(V_{0}\right)$ since $\operatorname{dim}\left(V_{0}\right)=n-1$ and $n>2$. Hence, $U=\{0\}$.

In Lemma 7.3, we found some $g \in G$ such that $\alpha_{S}(a g) \neq 0$. We calculate $\sum_{h \in G} \alpha_{S}(a h)$ as follows,

$$
\begin{aligned}
\sum_{h \in G} \alpha_{S}(a h) & =\sum_{h \in G}\left\langle S^{\prime},[Q] M_{a} M_{h}\right\rangle \\
& =\left\langle S^{\prime},[Q] M_{a}\left(\sum_{h \in G} M_{h}\right)\right\rangle \\
& =\left\langle S^{\prime},[Q]\left(M_{a}-I\right)\left(\sum_{h \in G} M_{h}\right)\right\rangle
\end{aligned}
$$


The last equality holds because

$$
\left\langle S^{\prime},[Q]\left(M_{a}-I\right)\left(\sum_{h \in G} M_{h}\right)\right\rangle=\left\langle S^{\prime},[Q] M_{a}\left(\sum_{h \in G} M_{h}\right)\right\rangle-\left\langle S^{\prime},[Q]\left(\sum_{h \in G} M_{h}\right)\right\rangle
$$

But, $[Q] M_{h}=[Q]$ for all $h \in G$, since $M_{h}$ is a permutation matrix. Therefore,

$$
\left\langle S^{\prime},[Q] \sum_{h \in G} M_{h}\right\rangle=\left\langle S^{\prime},|G|[Q]\right\rangle=0
$$

since $S^{\prime} \perp[Q]$.

By Lemma 7.6,

$$
[Q]\left(M_{a}-I\right)\left(\sum_{h \in G} M_{h}\right)=0
$$

since $[Q]\left(M_{a}-I\right) \in V_{0}$ by Lemma 5.5 . Thus,

$$
\sum_{h \in G} \alpha_{S}(a h)=0
$$

But, we already found some $g \in G$ such that $\alpha_{S}(a g) \neq 0$. To maintain the zero sum in (7.6), not all the $\alpha_{S}(a g)$ can be negative and so there exists $g^{\prime} \in G$ such that $\alpha_{S}\left(a g^{\prime}\right)>0$. This implies that

$$
\left|S\left(a g^{\prime}\right)^{-1}\right|-|S|>0
$$

and hence,

$$
\left|S\left(a g^{\prime}\right)^{-1}\right|>|S|
$$

Thus, if $u \in A^{*}$ represents $a g^{\prime}$, then $\left|S u^{-1}\right|>|S|$. We conclude, since $S$ was arbitrary, that there must be a synchronizing word for $(Q, A)$ as we saw in Chapter 2

To bound the size of a synchronizing word, according to Chapter 2, we must bound the length of $u$. For each $g \in G$, choose $U_{g} \in A^{*}$ to be a minimal length word representing $g$. Let $m=\max \left\{\left|U_{g}\right| \mid g \in G\right\}$. Then $a g^{\prime}$ can be represented by $a U_{g^{\prime}}$, which has length at most $m+1$. The strategy in Chapter 2 shows that $(Q, A)$ has a synchronizing word of length at most $1+(n-2)(m+1)$.

We have thus proved the following result. 
Theorem 7.7 (Steinberg). Let $(Q, A)$ be an automaton and $P \subseteq A$ be a set of permutations (not all trivial) such that $V_{0}$ is $G$-irreducible with $G=\langle P\rangle$. Let $m$ be the maximum length of a minimal length representative from $A^{*}$ of an element $G$. If A contains a non-permutation, then $(Q, A)$ is synchronizing and a synchronizing word can be found of length at most $1+(n-2)(m+1)$.

COROllary 7.8 (Pin $[\mathbf{1 5}])$. Let $(Q, A)$ be a circular automaton on $n$ states with $n$ prime. Suppose $A$ contains a non-permutation. Then $(Q, A)$ is synchronizing and has a synchronizing word of length at most $(n-1)^{2}$.

ProOF. Suppose without loss of generality that

$$
p=\left(\begin{array}{cccc}
1 & 2 & \ldots & n \\
2 & 3 & \ldots & 1
\end{array}\right) \in A
$$

and let $G=\langle p\rangle$. Then $G=\left\{p^{0}, p^{1}, \ldots, p^{n-1}\right\}$. By Proposition 7.1, $V_{0}$ is $G$-irreducible. But, $m \leq n-1$ since each element of $G$ has a representative of length at most $n-1$. Therefore, there is a synchronizing word of length at most

$$
1+(n-2) n=1+n^{2}-2 n=(n-1)^{2}
$$

by Theorem 7.7 .

EXAMPLE 7.9. We construct an Irreducible Permutation Automaton as follows,

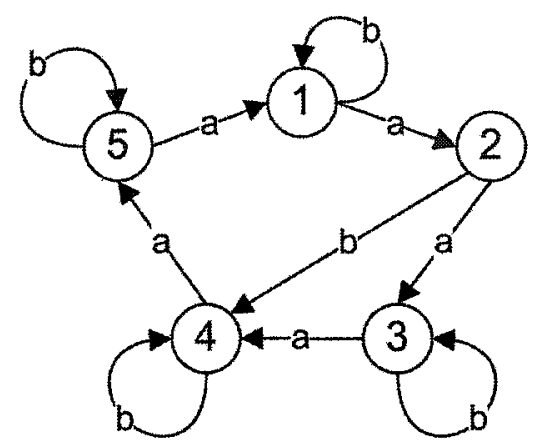

For this example, a synchronizing word is $b a^{3} b a^{3} b a^{3} b$. This is clearly shortest since we need 4 bs. Also, using a similar argument to the one we used in Theorem 2.7, we see that we will need 3 as between each pair of bs. 
EXAMPLE 7.10. For $S_{n}$, with $P=\left\{\left(\begin{array}{ll}1 & 2\end{array}\right),(23), \ldots,(n-1 n)\right\}, m \leq n[10]$. Thus, by applying Theorem 7.7, we see that a synchronizing word has length at most $1+(n-2)(n+1)=n^{2}-n-1$. This could also be proved using the Exterior Power automaton. 


\section{CHAPTER 8}

\section{Conclusion}

Cernýs Conjecture says that every synchronizing automaton with $n$ states has a shortest synchronizing word with length at most $(n-1)^{2}$. In this paper, we considered two different approaches to this problem. We used a combinatorial approach for Černýs examples, the Exterior Power automaton, and Weakly Orientable automata. Then, we used a unified linear algebra approach for the Eulerian automata and Irreducible Permutation automata. Each approach has its advantages and disadvantages. The combinatorial approach offers conceptually easier proofs since we can draw diagrams for illustration but the proofs tended to be very specific to the type of automata being considered. In contrast, the linear algebra offers easier proofs since we could often just manipulate equations to get meaningful results. Moreover, this approach seems to be more robust and perhaps more powerful $[\mathbf{6}]$. The disadvantage is that we aren't able to draw diagrams. 


\section{Bibliography}

1. D. S. Ananichev, M. V. Volkov, Some results on Černý type problems for transfomation semigroups in "Proceedings Of The Workshop: Semigroups and Languages" I. M. Araújo, M. J. J. Branco, V. H. Fernandes, G. M. S. Gomes (Eds.), World Scientific, Singapore, (2004), pp. 23-42.

2. Y. Benenson, T. Paz-Elizur, R. Adar, E. Keinan, Z. Livneh, E. Shapiro, Programmable and autonomus computing machines made of biomolecules, Nature 414, (2001), pp. 430-434.

3. Y. Benenson, R. Adar, T. Paz-Elizur, Z. Livneh, E. Shapiro, DNA molecule provides a computing machine with both data and fuel, Proceedings of the National Academy of Sciences USA 100 (2003), pp. 2191-2196.

4. J. Berstel and C. Reutenauer, "Rational Series and Their Languages", EATCS Monographs on Theoretical Computer Science, 12. Springer-Verlag, Berlin, (1988).

5. J. Černý, Poznámka k homogénnym eksperimentom s konecnými avtomatami, Mat.-Fyz. Cas. Solvensk. Akad. Vied. 14 (1964), pp. 208-216 [in Slovak].

6. L. Dubuc, Sur les automates circulaires et la conjecture de Černý. [Circular automata and Cerny's conjecture], RAIRO Inform. Thor. Appl. 32 (1998), pp. 21-34.

7. D. Eppstein, Reset sequences for monotonic automata, SIAM J. Comput. 19 (1990), pp. 500-510.

8. J. Kari, Synchronizing finite automata on Eulerian digraphs, Mathematical foundations of computer science (Mariánské Lázně, (2001). Theoret. Comput. Sci. 295 (2003), pp. 223-232.

9. P. Linz, "An Introduction To Formal Languages And Automata - 3rd edition", Jones and Bartlett, Sudbury, Massachusetts, (2001).

10. M. Hall, "The Theory of Groups", MacMillan, New York, (1959).

11. B. K. Natarjan, An algorithmic approach to the automated design of parts orienters, Proceedings of the 27th Annual Symposium Foundations of Computer Science, IEEE, (1986), pp. 132-142.

12. B. K. Natarjan, Some paradigms for automated design of part feeders, International Journal of Robotics Research, 8, (1989), pp. 89-109.

13. W. K. Nicholson, "Introduction to Abstract Algebra", John Wiley \& Sons, New York, (1999).

14. M. S. Paterson, Unsolvability in $3 \times 3$ matrices, Studies in Appl. Math. 49 (1970), pp. 105-107. 
15. J.-E. Pin, On two combinatorial problems arising from automata theory, Annals of Discrete Mathematics 17 (1983), pp. 535-548.

16. A. Salomaa, Composition sequences of functions over a finite domain, Theoretical Computer Science 292 (2003), pp. 263-281.

17. A. Salomaa, Synchronization of finite automata: contributions to an old problem, in "The Essence of Computation. Complexity, Analysis, Transformation: Essays Dedicated to Neil D. Jones" T. Mogensen, D.A. Schmidt, I. Hal Sudborough (Eds.), Springer LNCS Volume 2566, (2002), pp. $37-59$. 